\title{
INCIDENCIA Y FACTORES DE RIESGO DE INFECCIONES DEL TORRENTE SANGUÍNEO ASOCIADAS A CATÉTER CENTRAL
}

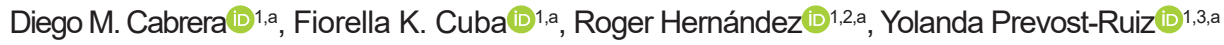 \\ 1 Facultad de Medicina Humana «Alberto Hurtado», Universidad Peruana Cayetano Heredia, Lima, Perú. \\ 2 Servicio de Infectología Pediátrica, Hospital Cayetano Heredia, Lima, Perú. \\ 3 Servicio de UCI Neonatal, Hospital Cayetano Heredia, Lima, Perú. \\ Médico cirujano
}

\section{RESUMEN}

Las infecciones del torrente sanguíneo asociadas a catéter central (ITSACC) generan gran morbimortalidad y elevados costos hospitalarios. Estudios sobre su incidencia y comportamiento clínico-epidemiológicos en la población neonatal en el Perú son escasos y no está del todo claro su impacto actual. Se realizó un estudio de cohorte prospectivo durante un año (2017-2018) en la unidad de cuidados intensivos (UCI) neonatales de un hospital público de Lima, Perú. Se incluyeron 167 pacientes (52,7\% varones) con edades gestacionales entre las 24-41 semanas, se registraron 1999 días-catéter y 16 casos de ITSACC. La incidencia fue de 8/1000 días-catéter. El uso de catéter umbilical $(\mathrm{p}=0,005)$ y el uso de múltiples catéteres $(\mathrm{p}<0,001)$ mostraron relación estadísticamente significativa respecto al desarrollo de ITSACC. Es necesario ampliar el estudio a otras UCI y establecer sistemas eficientes y duraderos de monitoreo que permitan la evaluación de intervenciones para reducir las ITSACC.

Palabras clave: Infecciones Relacionadas con Catéteres; Recién Nacido; Factores de Riesgo; Perú (fuente: DeCS BIREME).

\section{INCIDENCE AND RISK FACTORS OF CENTRAL LINE CATHETER-RELATED BLOODSTREAM INFECTIONS}

\begin{abstract}
Central line catheter-related bloodstream infections (CLABSI) burdens great morbidity, mortality and unnecessary hospital expenses. Studies related to its incidence and epidemiological and clinical profile among neonates in Peru are scarce, not being clear it's actual impact. A prospective cohort study was conducted in a neonatal intensive care unit (ICU) of a public hospital in Lima, Peru between 20172018. 167 patients were included (52,7\% male) with gestational age between 24-41 weeks, obtaining 1999 catheter-days and 16 cases of CLABSI. The incidence rate was 8/1000 catheter-days. Use of umbilical catheter $(\mathrm{p}=0,005)$ and multiple catheters $(\mathrm{p}<0,001)$ both showed a statistically significant correlation regarding the development of CLABSI. It's necessary to extend the study to other ICUs and stablish solid, efficient and long-lasting system of CLABSI surveillance that allows the evaluation of possible interventions to reduce the incidence of CLABSI.
\end{abstract}

Keywords: Catheter-Related Infections; Newborn; Risk Factors; Peru (source: MeSH NLM).

Citar como: Cabrera DM, Cuba FK, Hernández R, Prevost-Ruiz Y. Incidencia y factores de riesgo de infecciones del torrente sanguíneo asociadas a catéter central. Rev Peru Med Exp Salud Publica. 2021;38(1):95-100. doi: https://doi.org/10.17843/ rpmesp.2021.381.5108.

Correspondencia: Diego Cabrera Chávez; Av. Honorio Delgado 430, San Martín de Porres;

diego.cabrera.c@upch.pe

Recibido: $16 / 01 / 2020$

Aprobado: $10 / 11 / 2020$

En línea: 01/02/2021

\section{INTRODUCCIÓN}

Las infecciones asociadas a la atención sanitaria (IAAS), o anteriormente llamadas infecciones intrahospitalarias (IIH), representan una gama de patologías que causan altas tasas de morbilidad y mortalidad. Estos eventos son más comunes en áreas críticas con poblaciones vulnerables, como una unidad de cuidados intensivos neonatales (UCIN), donde los pacientes son propensos a prolongadas estancias hospitalarias y procedimientos invasivos, como la inserción de catéteres (centrales y periféricos). El uso de catéteres centrales en el neonato corresponde a una práctica común en el manejo médico ${ }^{(1)}$. No obstante, al ser un dispositivo invasivo, genera una puerta de ingreso para diversos microorganismos. Además, tanto la colocación como el mantenimiento del mismo han sido asociados a casos de sobreinfección ${ }^{(2)}$.

En el Perú, reportes epidemiológicos describen importantes medidas de frecuencia de IAAS durante las últimas décadas. En el 2013, se describe que el 12,7\% de las 9812 IAAS per- 
tenecían al servicio de neonatología ${ }^{(3)}$. De la misma manera, en el 2018, se reporta una tasa de incidencia de infecciones del torrente sanguíneo asociada a catéter central (ITSACC) de 4,92 , siendo mayor a otros tipos de IAAS reportadas en dicho año ${ }^{(3)}$.

El impacto de las ITSACC es importante tanto para el pronóstico del paciente como para la salud pública ${ }^{(4,5)}$. Warren et al. reportaron que los costos hospitalarios totales en neonatos que han padecido esta patología casi triplican a los de neonatos que no fueron afectados ${ }^{(4)}$. No obstante, se reconoce que intervenciones preventivas pueden aminorar estas complicaciones ${ }^{(6)}$. Para ello, se necesitan estudios longitudinales que exploren la epidemiología de dicha enfermedad. El objetivo del presente estudio es determinar la incidencia de las ITSACC en la UCIN de un hospital público en Lima, Perú y explorar los factores de riesgo asociados a dicha patología.

\section{EL ESTUDIO}

\section{Diseño y participantes de estudio}

Se realizó una cohorte prospectiva de pacientes admitidos en la UCIN del Hospital Cayetano Heredia $(\mathrm{HCH})$ entre junio del 2017 y junio del 2018. El HCH es un hospital público que cuenta con una UCIN de 10 camas y responde a una importante demanda de neonatos anualmente. Al ser el presente estudio una cohorte abierta, se incluyeron a todos los neonatos que cumplieran con los criterios de elegibilidad durante el periodo mencionado, no hubo un cálculo formal de tamaño de muestra, se trabajó con el universo poblacional. Se incluyeron a todos los neonatos admitidos en la UCIN del HCH que portaron un catéter central por al menos 48 horas. Se consideró como catéter central a aquel que termina localizado en el corazón o en un vaso principal aledaño a éste, independiente de la zona de inserción, ya sea catéter umbilical arterial (CUA), catéter umbilical venoso (CUV) o catéter central insertado periféricamente (PICC, por sus siglas en inglés). Se excluyó a pacientes transferidos de otras instituciones de salud que portaran dichos tipos de catéter al momento del ingreso.

\section{Variables}

Se consideró como variable dependiente a la ITSACC definida según los criterios del Centers for Disease Control and Prevention (CDC) como aquella infección en un paciente que porta un catéter central por más de 2 días-calendario al día del evento, donde el día de inserción del dispositivo es el «día 1»y el catéter está implantado el día del evento o el día anterior ${ }^{(7)}$. El seguimiento fue desde la colocación del catéter central (día 1) hasta la aparición del evento (aparición de los primeros signos clínicos de infección). El cuadro clínico compatible con infección fue evaluado, en cada turno, por el equipo médico de la UCIN que sigue un protocolo establecido ante la sospecha

\section{MENSAJES CLAVE}

Motivación para realizar el estudio: Las infecciones asociadas a catéter generan una gran carga para los sistemas de salud y los pacientes que las padecen. En Perú, estudios sobre su incidencia y comportamiento en neonatos son escasos y no está del todo claro su impacto actual.

Principales hallazgos: Se encontró una incidencia de infección de 8/1000 días-catéter, superior a reportes nacionales e internacionales previos. Los factores asociados fueron el uso de catéter umbilical como factor protector y el uso de múltiples catéteres como factor de riesgo.

Implicancias: Es necesario establecer programas eficientes y duraderos de monitoreo de infecciones intrahospitalarias que permitan el planteamiento y evaluación de intervenciones preventivas.

de infección, el cual incluye la evaluación de signos clínicos y la analítica correspondiente (hemograma, PCR, cultivos) descritos por Haque ${ }^{(8)}$. La sospecha de infección fue confirmada posteriormente con un hemocultivo y cultivo de catéter positivo por el área de microbiología del $\mathrm{HCH}$ siguiendo procedimientos estandarizados ${ }^{(9)}$. Aquellos que permanecieron asintomáticos o presentaron clínica sugestiva de infección que no fue confirmada por cultivos fueron seguidos hasta el retiro del catéter, muerte o alta de la unidad.

Como variables independientes, se recolectaron datos antropométricos como el peso (en gramos), edad gestacional (EG) (en semanas) y el sexo, siguiendo las definiciones establecidas por la Organización Mundial de la Salud (OMS) ${ }^{(10)}$. Se recolectaron datos relacionados al catéter central, como el lugar de inserción (CUA, CUV, PICC), uso de nutrición parenteral total (NPT), días de utilización (días-catéter) y uso de dos a más catéteres en simultáneo. Se registró el hacinamiento como una variable de control, definida como la atención de pacientes superior al cupo permitido en la UCIN (>10 camas), en donde no existe una separación mínima de dos metros lineales entre dos camas ${ }^{(11)}$.

\section{Instrumentos y recolección de información}

Se utilizaron dos fichas digitalizadas con una interfaz en $\mathrm{Mi}$ crosoft Access $\odot$. Ambas fichas fueron previamente validadas por el servicio de la UCIN, teniendo en cuenta factores de riesgo reportados previamente en la literatura ${ }^{(12,13)}$. Las mediciones fueron obtenidas mediante procedimientos estandarizados, utilizando un mismo instrumento para todos los participantes, los datos referentes al catéter fueron obtenidos de la historia clínica del paciente. Con la primera ficha 
se recolectó información referente a datos antropométricos, nutrición, y dispositivos utilizados en el paciente, dentro de ello, datos relacionados a la colocación y mantenimiento de catéteres. La segunda ficha permitió recolectar la información de los pacientes con sospecha de infección e incluía datos clínicos, analítica de laboratorio y cultivos. Si un paciente con sospecha de infección no tenía una infección asociada a catéter, regresaba a la cohorte aportando días de exposición.

\section{Análisis estadístico}

Se utilizaron medidas de frecuencia, medias, desviación estándar, medianas y rangos intercuartiles para describir las variables independientes. La asociación entre los factores de riesgo y la ITSACC fue analizada mediante pruebas de T de Student y $\mathrm{U}$ de Mann Whitney para las variables cuantitativas; chi cuadrado y prueba de proporciones para las variables cualitativas. Se realizó una regresión univariante con análisis de Poisson para explorar el riesgo relativo (RR) entre los factores de riesgo previamente reportados en la literatura (uso de catéter umbilical, días-catéter, peso, hacinamiento, NPT, uso de múltiples catéteres) y la ITSACC previo análisis de normalidad de las variables cuantitativas. La tasa de incidencia de ITSACC fue realizado por medio de la división entre el número de infecciones asociadas al dispositivo y la suma del total de días-catéter. Se utilizó el paquete estadístico STATA 15.0 (StataCorp, Texas, EE. UU.). Se consideró un valor de $\mathrm{p}<0,05$ como estadísticamente significativo.

\section{Consideraciones éticas}

La investigación fue aprobada por los comités de ética de la Universidad Peruana Cayetano Heredia (155-08-19) y del $\mathrm{HCH}$. La identidad de los participantes fue protegida mediante la asignación de códigos numéricos en la base de datos. Al no interactuar directamente con los participantes, no se requirió el uso de consentimiento informado.

\section{HALLAZGOS}

Se incluyeron 167 neonatos, en los cuales fueron colocados 322 catéteres centrales en 1999 días-catéter. El 47,3\% fue del sexo femenino y la media de la EG fue de 32,3 (DE: 4) vs. 34,6 (DE: 3,5) semanas entre el grupo ITSACC y no ITSACC $(\mathrm{p}<0,05)$. El $82 \%$ fueron prematuros ( $\leq 37$ semanas) y la mediana de peso al momento de la inserción del catéter fue de 1990 gramos (Tabla 1).

Se diagnosticaron 16 casos de ITSACC $(9,6 \%$ de la cohorte total). Con ello, la tasa de incidencia fue de 8/1000 días-catéter. Todos los casos de ITSACC fueron en prematuros y el 56,3\% de ellos presentó un peso inferior a 1500 gramos. El 50\% (8/16) fueron mujeres. Se aislaron bacterias grampositivas en el $62,5 \%$ y gramnegativas en el $37,5 \%$.
Entre todos, el Staphylococcus aureus fue la bacteria más frecuente $(31,3 \%)$ (Tabla 2). Respecto a las variables relacionadas al catéter, el 93,8\% de participantes con ITSACC portaron múltiples catéteres en algún momento de su estadía (50\%, 3 catéteres; $43,8 \%, 2$ catéteres) y la indicación más frecuente fue NPT (81,3\%). Respecto al lugar de inserción del catéter, el $66,7 \%$ de los catéteres asociados a ITSACC fueron colocados por vía umbilical y el 33,3\% por vía periférica. La variable de control hacinamiento estuvo presente en algún momento de la estancia hospitalaria en el 62,5\% de los casos.

En el análisis bivariado, el uso de catéter umbilical (RR: 0,25; IC95\%: 0,09-0,65; $\mathrm{p}=0,005)$ y el uso de múltiples catéteres (RR: 4,98; IC95\%: 2,05-12,12; $\mathrm{p}<0,001$ ) mostraron ser factores estadísticamente significativos respecto al desarrollo de ITSACC. El sexo femenino, mayor días-catéter, presencia de hacinamiento y el uso de NPT mostraron un incremento de riesgo, pero no estadísticamente significativo (Tabla 3).

\section{DISCUSIÓN}

La ITSACC es un evento adverso de la atención sanitaria cuya magnitud a nivel nacional se conoce parcialmente, en base a la vigilancia epidemiológica del Ministerio de Salud (MINSA). Sin embargo, a nivel hospitalario se desconoce con exactitud su incidencia. Este estudio propone cubrir una brecha en el conocimiento, estimando en una cohorte prospectivamente enrolada en un año, la magnitud de la ITSACC. La tasa de incidencia de ITSACC en neonatos admitidos en la UCIN de un hospital público peruano fue de 8/1000 días-catéter, incidencia mayor a la descrita a nivel nacional e internacional ${ }^{(3,14)}$.

Coincidiendo con diversos estudios, los microorganismos encontrados fueron Staphylococcus aureus, seguido del Staphylococcus coagulasa negativo ${ }^{(15)}$. Ambos, frecuentes colonizadores de la piel, principal puerta de entrada en esta condición ${ }^{\left({ }^{16}\right)}$. Asimismo, solo el 37,5\% de los casos se dieron por bacterias gramnegativas. Ello concuerda con estudios realizados por la National Healthcare Safety Network en Estados Unidos entre el 2016-2017 en donde se reportan tasas bajas de infección por estas bacterias ${ }^{(7)}$. El bajo porcentaje de casos asociados a bacterias gramnegativas coincide con la mayor elección de la cabeza y miembros superiores como sitio de inserción del dispositivo en lugar de la inserción femoral. Esta localización es la de mayor riesgo por su cercanía a la flora urinaria y fecal ${ }^{(17)}$.

Entre los factores asociados a la ITSACC, la edad gestacional menor a 28 semanas y un peso menor a 1500 gramos al momento de la inserción del catéter son señalados como factores de riesgo importantes ${ }^{(18)}$. Coincidiendo con 
Tabla 1. Características clínicas de las infecciones del torrente sanguíneo asociada a catéter central en la unidad de cuidados intensivos neonatal de un hospital público de nivel III-1.

\begin{tabular}{|c|c|c|c|c|}
\hline \multirow[t]{2}{*}{ Características clínicas } & $\begin{array}{c}\text { Cohorte total } \\
(\mathrm{N}=167)\end{array}$ & $\begin{array}{c}\text { Grupo ITSACC } \\
(n=16)\end{array}$ & $\begin{array}{c}\begin{array}{c}\text { Grupo No ITSACC } \\
(n=151)\end{array} \\
\end{array}$ & \multirow[t]{2}{*}{ Valor de $\mathbf{p}^{\mathrm{d}}$} \\
\hline & $\mathrm{N}(\%)$ & n (\%) & n (\%) & \\
\hline \multicolumn{5}{|l|}{ Sexo } \\
\hline Masculino & $88(52,7)$ & $8(50,0)$ & $80(52,9)$ & $0,872^{\mathrm{e}}$ \\
\hline Femenino & $79(47,3)$ & $8(50,0)$ & $71(47,1)$ & \\
\hline Edad gestacional ${ }^{\text {a }}$ & $34,40(3,6)$ & $32,3(4,0)$ & $34,61(3,5)$ & $0,017^{\mathrm{f}}$ \\
\hline$\leq 37$ semanas & $137(82,1)$ & $16(100,0)$ & $121(80,1)$ & 0,049 \\
\hline$>37$ semanas & $30(17,9)$ & $0(0,0 \%)$ & $30(19,9)$ & - \\
\hline Peso (gramos) ${ }^{b}$ & $1990(1380-2905)$ & $1415,5(1183,5-2455)$ & $2078(1458-2990)$ & $0,028^{g}$ \\
\hline$<1000$ & $10(5,9)$ & $2(12,5)$ & $8(5,3)$ & 0,716 \\
\hline $1000-1499,9$ & $40(23,9)$ & $7(43,8)$ & $33(21,9)$ & 0,228 \\
\hline $1500-2499,9$ & $55(32,9)$ & $3(18,7)$ & $52(34,4)$ & 0,576 \\
\hline$>2500$ & $62(37,3)$ & $4(25,0)$ & $58(38,4)$ & 0,592 \\
\hline Días-catéter a & $11,97(7,6)$ & $12,25(5,6)$ & $11,94(7,8)$ & $0,878^{\mathrm{f}}$ \\
\hline \multicolumn{5}{|l|}{ Localización del catéter ${ }^{c}$} \\
\hline CUA & $133(79,6)$ & $15(93,8)$ & $118(78,1)$ & 0,153 \\
\hline CUV & $110(65,9)$ & $11(37,5)$ & $99(65,6)$ & 0,067 \\
\hline PICC MMSS & $62(37,1)$ & $6(37,5)$ & $56(37,1)$ & 0,980 \\
\hline PICC MMII & $10(5,9)$ & $6(37,5)$ & $4(2,6)$ & 0,202 \\
\hline PICC cabeza & $7(4,2)$ & $1(6,3)$ & $6(3,9)$ & - \\
\hline
\end{tabular}

${ }^{a}$ Media (desviación estándar)

${ }^{\mathrm{b}}$ Mediana (rango intercuartílico)

' Participantes utilizaron más de un tipo de catéter al mismo tiempo durante el reclutamiento, por lo que se reporta el número total de catéteres utilizados por cada categoría

¿ Prueba de proporciones

e Prueba de chi cuadrado

f Prueba T de Student

${ }^{g}$ Prueba U de Mann Whitney

ITSACC: infección del torrente sanguíneo asociada a catéter central; CUA: catéter umbilical arterial; CUV: catéter umbilical venoso; PICC: catéter central insertado periféricamente; MMSS: miembros superiores; MMII: miembros inferiores.

lo reportado, respecto a los casos de ITSACC, el 100\% presentó una edad gestacional menor a 37 semanas, aunque no se encontró relación estadísticamente significativa respecto al peso y la ocurrencia de ITSACC; pese a que ambos factores se interrelacionan con gran frecuencia y representan

Tabla 2. Microorganismos aislados en casos de infección del torrente sanguíneo asociada a catéter central en la unidad de cuidados intensivos neonatal de un hospital público de nivel III-1.

\begin{tabular}{lc}
\hline Microorganismos & n (\%) \\
\hline Grampositivo & $10(62,5)$ \\
Staphylococcus aureus & $5(31,3)$ \\
Staphylococcus epidermidis & $4(25)$ \\
Staphylococcus haemolyticus & $1(6,3)$ \\
Gramnegativo & $6(37,5)$ \\
Pseudomona aeruginosa & $2(12,5)$ \\
Klebsiella spp. & $2(12,5)$ \\
Klebsiella pneumoniae & $1(6,3)$ \\
Stenotrophomonas spp. & $1(6,3)$ \\
\hline
\end{tabular}

condiciones severas que requieren mayor manipulación del catéter, hecho que se ha asociado a bacteriemia ${ }^{(13)}$.

Respecto a las variables relacionadas al catéter, el uso múltiple y la localización de inserción umbilical, se reportan como factores predisponentes de infección ${ }^{(15,18,19)}$. En el aná-

Tabla 3. Análisis bivariado entre las variables independientes y la infección del torrente sanguíneo asociada a catéter central en la unidad de cuidados intensivos neonatal de un hospital público de nivel III-1.

\begin{tabular}{lccc}
\hline \multirow{2}{*}{ Variables independientes } & \multicolumn{2}{c}{ Análisis Bivariado } & \multirow{2}{*}{ Valor de p } \\
\cline { 2 - 3 } & $\mathbf{R R}$ & IC 95\% & \\
\hline Características clínicas & & & \\
Sexo femenino & 1,11 & $0,42-2,96$ & 0,829 \\
Uso de catéter umbilical & 0,25 & $0,09-0,65$ & 0,005 \\
Días-catéter & 1,00 & $0,94-1,07$ & 0,883 \\
Peso (gramos) & 0,99 & $0,99-0,99$ & 0,043 \\
Hacinamiento & 2,61 & $0,95-7,19$ & 0,063 \\
NPT & 2,25 & $0,64-7,87$ & 0,207 \\
Uso de múltiples catéteres & 4,98 & $2,05-12,12$ & $<0,001$ \\
\hline NPT: nutrición parenteral total; RR: riesgo relativo; IC 95\%: intervalos de confianza al 95\%.
\end{tabular}


lisis bivariado, se encontró que el uso de múltiples catéteres presenta una relación estadísticamente significativa con la presencia de ITSACC. Sin embargo, contrario a la literatura, la inserción umbilical fue un factor protector, posiblemente por el corto tiempo de uso en comparación con los PICC.

En cuanto al hacinamiento, medida que refleja la capacidad de un hospital de cuidar adecuadamente a sus pacientes ${ }^{(20)}$. Se encontró que 62,5\% del grupo con ITSACC estuvo en un contexto de hacinamiento durante algún momento de su hospitalización. No obstante, no llegó a ser estadísticamente significativo en el modelo bivariado. Causas previamente reportadas son la menor disponibilidad de personal por paciente y con ello, una potencialmente reducida aplicación de medidas de prevención de infecciones ${ }^{(20)}$.

Entre las limitaciones del estudio está la ausencia de un cálculo de tamaño de muestra y el uso de un análisis bivariado no ajustado, lo que podría haber afectado el análisis de las variables; y la naturaleza monocéntrica que afecta la extrapolación a otros contextos. Si bien, hubo dificultad para cumplir estrictamente con el criterio microbiológico en el diagnóstico de la ITSACC debido a la falta de equipos y personal, se utilizó los criterios indicados por el CDC y el MINSA, usados principalmente para propósitos de vigilancia epidemiológica.

\section{REFERENCIAS BIBLIOGRÁFICAS}

1. Oliveira Gomes AV, Luca Nascimento MA. Central venous catheterization in Pediatric and Neonatal Intensive Care Units. Rev Esc Enferm USP. 2013;47(4):794-800. doi: 10.1590/S0080-623420130000400004.

2. Castro López F. Beneficios del catéter epicutáneo en el recién nacido. Rev Cubana Enfermer. 2004;20(2):1-1.

3. Quispe Z. Situación de las infecciones asociadas a la atención en salud, perú -2016. Dirección General de Epidemiología. Published 2016 (Acceso 14 diciembre 2019). Disponible en: http://www.dge.gob.pe/ portal/docs/tools/teleconferencia/SE102017/02.pdf.

4. Warren DK, Quadir WW, Hollenbeak CS, Elward AM, Cox MJ, Fraser VJ. Attributable cost of catheter-associated bloodstream infections among intensive care patients in a nonteaching hospital. Crit Care Med. 2006;34(8):2084-2089. doi:10.1097/01.CCM.0000227648.15804.2D.

5. Estimating the Additional Hospital Inpatient Cost and Mortality Associated With Selected Hospital-Acquired Conditions | Agency for Health Research and Quality (Acceso 18 diciembre 2019). Disponible en: https://www.ahrq.gov/hai/pfp/haccost2017-results.html.

6. Bell T, O'Grady NP. Prevention of Central Line-Associated Bloodstream Infections. Infect Dis Clin North Am. 2017;31(3):551-559. doi:10.1016/j.idc.2017.05.007.

7. Haddadin Y, Regunath H. Central Line Associated Blood Stream Infections (CLABSI). In: StatPearls. StatPearls Publishing; 2019 (Acceso 14 diciembre 2019). Disponible en: http://www.ncbi.nlm.nih.gov/books/ NBK430891/.

8. Haque KN. Neonatal Sepsis in the Very Low Birth Weight Preterm Infants: Part 2: Review of Definition, Diagnosis and Management. J Med Sci. 2010;3(1):11-27.

9. Hernandez L, Amaro C. Procedimiento Operacional Estándar Del Sistema de Gestión de Calidad Para Toma de Muestra Para Hemocultivo.
En conclusión, la incidencia de la ITSACC fue de 8/1000 días-catéter, cifra mayor a reportes previos. Dicho evento adverso acarrea una gran carga de morbi-mortalidad para los que la padecen. Por ello, se propone la realización de estudios prospectivos multicéntricos con mayor población que permitan la inferencia de otros factores de riesgo y la aplicación de estrategias de control de infecciones con posterior medición del impacto de estas intervenciones en la incidencia. Finalmente, si bien el número de casos es limitado, los hallazgos plantean el establecimiento de un programa de control de infecciones que permita reducir los factores de riesgo y disminuir la morbilidad.

Contribuciones de los autores: DMC y FKC contribuyeron en la concepción y diseño del estudio, redacción del protocolo, recolección, análisis e interpretación de datos, revisión y aprobación de la versión final del manuscrito. YPR contribuyó en la concepción y diseño del estudio, creación y diseño del protocolo, análisis e interpretación de datos, redacción y revisión crítica del manuscrito, aprobación de la versión final, asesoría técnica y estadística. RH contribuyó en el análisis e interpretación de datos, revisión crítica del manuscrito, aprobación de la versión final, asesoría técnica y estadística.

Financiamiento: Autofinanciado por los autores.

Conflicto de intereses: Ninguno.
Lima: Hospital Cayetano Heredia; 2016. Disponible en: http://www. hospitalcayetano.gob.pe/transparencia/images/stories/resoluciones/ RD/rd2016/rd_767_2016.pdf.

10. Cutland CL, Lackritz EM, Mallett-Moore T, Bardají A, Chandrasekaran R, Lahariya C, et al. Low birth weight: Case definition \& guidelines for data collection, analysis, and presentation of maternal immunization safety data. Vaccine. 2017;35(48Part A):6492-6500. doi: 10.1016/j. vaccine.2017.01.049.

11. Ministerio de Salud. Norma técnica de los servicios de cuidados intensivos de los hospitales del sector salud. Lima: MINSA; 2004. Disponible en: ftp://ftp2.minsa.gob.pe/destacados/archivos/46/ Norma\%20T\%E9cnica\%20Unidad\%20Cuidados\%20Intensivos.pdf.

12. García H, Romano-Carro B, Miranda-Novales G, González-Cabello HJ, Núñez-Enríquez JC. Risk Factors for Central Line-Associated Bloodstream Infection in Critically Ill Neonates. Indian J Pediatr. 2019;86(4):340-46. doi:10.1007/s12098-019-02896-6.

13. Mahieu LM, De Muynck AO, Ieven MM, De Dooy JJ, Goossens HJ, Van Reempts PJ. Risk factors for central vascular catheter-associated bloodstream infections among patients in a neonatal intensive care unit. J Hosp Infect. 2001;48(2):108-116. doi:10.1053/jhin.2001.0984.

14. Dudeck MA, Edwards JR, Allen-Bridson K, Gross C, Malpiedi PJ, Peterson KD, et al. National Healthcare Safety Network report, data summary for 2013, Device-associated Module. Am J Infect Control. 2015;43(3):206-221. doi: 10.1016/j.jijic.2014.11.014.

15. Dubbink-Verheij GH, Bekker V, Pelsma ICM, van Zwet EW, Smits-Wintjens VEHJ, Steggerda SJ, et al. Bloodstream Infection Incidence of Different Central Venous Catheters in Neonates: A 
Descriptive Cohort Study. Front Pediatr. 2017;5:142. doi:10.3389/ fped.2017.00142.

16. Guía de Practica Clínica Prevención, diagnóstico y tratamiento de las infecciones relacionadas a lineas vasculares. México, D. F: Instituto Secretaría de Salud; 2012. (Acceso 14 diciembre 2019). Disponible en: http://www.cenetec.salud.gob.mx/descargas/gpc/CatalogoMaestro/ IMSS_273_13_INFECCIONLINEASVASCULARES/273GER.pdf.

17. Hussain R, Cevallos ME, Darouiche RO, Trautner BW. Gram-negative intravascular catheter-related bacteremia in patients with spinal cord injury. Arch Phys Med Rehabil. 2008;89(2):339-342. doi:10.1016/j. apmr.2007.08.151.
18. Yumani DFJ, van den Dungen FAM, van Weissenbruch MM. Incidence and risk factors for catheter-associated bloodstream infections in neonatal intensive care. Acta Paediatr. 2013;102(7):e293-298. doi:10.1111/ apa.12256.

19. Concannon C, van Wijngaarden E, Stevens V, Dumyati G. The effect of multiple concurrent central venous catheters on central line-associated bloodstream infections. Infect Control Hosp Epidemiol. 2014;35(9):1140-1146. doi:10.1086/677634.

20. Kaier K, Mutters NT, Frank U. Bed occupancy rates and hospital-acquired infections--should beds be kept empty?. Clin Microbiol Infect. 2012;18(10):941-945. doi:10.1111/j.1469-0691.2012.03956.x. 